\title{
The Research of Overdriving for Liquid Crystal Spatial Light Modulators
}

\author{
Hongyang Guo $0^{1,2,3, a}$, Shengping $\mathrm{Du}^{1,2, b}$
}

${ }^{1}$ Key Laboratory of Optical Engineering, Chinese Academy of Sciences,Chengdu 610209,China

${ }^{2}$ The Institute of Optics and Electronics, The Chinese Academy of Sciences,Chengdu 610209,China

3 University of Chinese Academy of Sciences,Beijing 100049, China

aguohongy93@163.com, bdu shengping@163.com

Keywords: liquid crystal; overdring; relaxation of liquid crystal; response speed

Abstract. Liquid crystal spatial light modulators are the core devices of the late-model beam deflection system, the slowly response speed is the key problem of the LCD engineering system. Based on the dynamic theory of liquid crystal, analyzing the relaxation properties and the factors influence the response time of liquid crystal, researching the overdriving principle, an overdriving method of liquid crystal based on FPGA is presented, which achieves rapid deflection of the liquid crystal molecules and improves the response speed. The results of simulation prove that choosing the right phase modulation period, driven by 5 voltage the increasing response time of $2 \pi$ reduced to 30 $\mathrm{ms}$, the fall response time of $2 \pi$ reduced to $70 \mathrm{~ms}$.

\section{引言}

液晶空间光调制器具有精确、灵巧、紧凑、保密性强等优点, 在航空航天、通信、光信息处 理与存储、生物医学和军事等诸多领域有着广泛的应用背景 ${ }^{[1]}$.液晶作为调制器控制系统的核 心器件, 其响应速度慢是影响液晶系统工程化的关键问题。目前, 提高液晶器件响应速度的

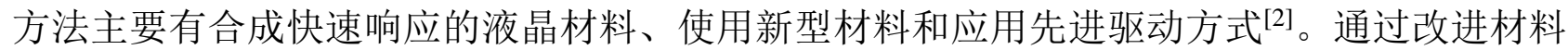
的方法可以使液晶空间光调制器的响应速度得到明显提高, 但潜力越来越有限, 过驱动方法 则可以在现有材料的基础上进一步提高响应速度，减小误差。

过驱动是指使用比正常驱动更大的电压差来驱动液晶分子，使其快速到达目标位相。该方 法最早由吴诗聪在1988年提出, 即瞬态向列效应 ${ }^{[3]}$ 。之后国内外对过驱动技术在显示领域的 应用开展大量的研究, 使得应用于显示领域的过驱动技术日益成熟, 但将过驱动应用于位相 调制器还很少见。本文提出一种基于FPGA的过驱动方案, 所有液晶过驱动过程均在FPGA中 实现，不占用CPU资源，更有利于液晶光束偏转等需要大量运算的场合。

\section{液晶的驰豫特性}

电场施加到液晶电极上后, 液晶分子偏转需要一定过程。对液晶盒施加电压经过无限长的时 间, 最终液晶分子的取向应由处于最低自由能状态决定, 这就是液晶分子的弛豫过程。一般 情况下液晶的弛豫时间达到 $10 \mathrm{~ms} 200 \mathrm{~ms}$, 弛豫过程包括介电弛豫和转向弛豫。液晶的介电弛 豫时间的典型值为 $10^{-4} \mathrm{~s} \sim 10^{-6} \mathrm{~s}$ ，对响应时间影响不大，所以重点考虑转向弛豫 ${ }^{[4]}$ 。

一般液晶忽略单个组成分子的存在, 将液晶看做连续介质来进行处理。液晶的指向矢的运动 可以用公式(1)Erickson-Leslie方程表征 ${ }^{[5]}$ :

$$
\left(E_{11} \sin ^{2} \theta+E_{a \mathrm{a}} \cos ^{2} \theta\right) \frac{\partial^{2} \theta}{\partial Z^{2}}+\left(K_{\mathrm{ag}}-K_{11}\right) * \sin \theta \cos \theta\left(\frac{\partial \theta}{\partial Z}\right)^{2}+\varepsilon_{0} \Delta \varepsilon E^{2} \sin \theta \cos \theta=-\gamma_{1} \frac{\partial \theta}{\partial t} .
$$

式中, $\gamma_{1}$ 为旋转粘度, $K_{\mathrm{ag}} 、 K_{11}$ 为弹性系数, $\varepsilon_{0} \Delta \varepsilon E^{2}$ 为外加电场强度, $\Delta \varepsilon$ 是介电各向异性常 数, $\theta$ 为指向矢与基板夹角, $z$ 为液晶分子在液晶盒中所处的位置。通常情况下, 忽略惯性效 应和回流效应, 在小角近似及单一弹性常数近似情况下，方程(1)可以化简为:

$$
K_{33} \frac{\mathrm{d}^{2} \theta}{\mathrm{d} z^{2}}+\varepsilon_{0} \Delta \varepsilon E^{2} \theta=-\gamma_{1} \frac{\partial \theta}{\partial t} .
$$


公式(2)的通解为:

$\theta=\theta_{m} \cos (\beta z) \cdot \exp (-t / \tau)$.

$\beta$ 为常数, $t$ 为液晶分子指向矢的响应时间。在给定的电压下, $\theta_{m}$ 代表液晶盒中央的最大倾 角 $\left(\left.\theta\right|_{z=0}=\theta_{m}\right), \tau$ 代表时间常数。当倾角 $\theta_{p}$ 为 0 的时针定能量很强, 可以得到边界条件:

$$
\left.\theta\right|_{z=-\frac{d}{2} \frac{d}{z}}=\theta_{p}=0
$$

由公式(3)、(4)可以得出液晶响应的上升时间常数公式(5)和下降时间常数公式(6)。

$$
\begin{aligned}
& \tau_{r}=\frac{\gamma_{1}}{\| s_{0}|\Delta s| E^{2}-\frac{\pi^{2}}{d^{2}} K_{s s} \mid}=\frac{\tau_{0}}{\left|\left(\frac{V}{V_{t h}}\right)^{2}-1\right|} . \\
& \tau_{d}=\tau_{0}=\frac{Y_{1 d^{2}}}{K_{s s \pi^{2}}} .
\end{aligned}
$$

式中 Vth为液晶的翻转阈值电压, $\mathrm{d}$ 为液晶盒厚度。可见, 减小液晶材料的粘滞系数或者 减小液晶盒厚度能降低弛豫时间, 但减小液晶盒厚度可能又会不满足其他电光特性。所以增 加驱动电压是更好的办法。

\section{过驱动原理及实现方法}

如图1所示, 虚线为在正常电压下液晶器件的响应过程, 在 $t_{3}$ 时刻才到达目标相位 $\varphi_{2}$ 。实线为 在过驱动条件下的相位响应, $t_{2}$ 时刻就可以到达相位 $\varphi_{2}$, 然后再切回正常电压, 相位将一直 保持在 $\varphi_{2}$, 相位从 $\varphi_{1}$ 到 $\varphi_{2}$ 的时间由原来的 $t_{3}$ 时刻缩短到 $t_{2}$ 时刻 ${ }^{[8]}$ 。

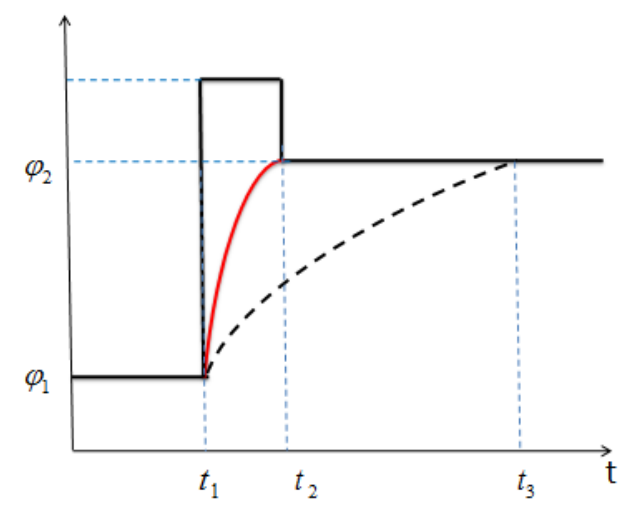

图1.过驱动原理

过驱动的实现就是对输入的电压进行处理后再输出新电压, 新电压使液晶分子偏转加快, 从而达到提高液晶响应速度的目的 ${ }^{[9]}$ 。如图 2 , 液晶驱动电路驱动引擎接受当前帧数据, 同时 从帧缓存器中读取前一帧数据, 将这两帧数据进行比较, 通过查取过驱动值表选取一个预设 定的过驱动量并输入到驱动电路, 实现驱动电压输入。在处理完当前帧的同时将该帧数据存 储在帧缓存器中来覆盖前一帧的数据, 用来处理下一帧的数据, 以此类推 ${ }^{[10]}$ 。 


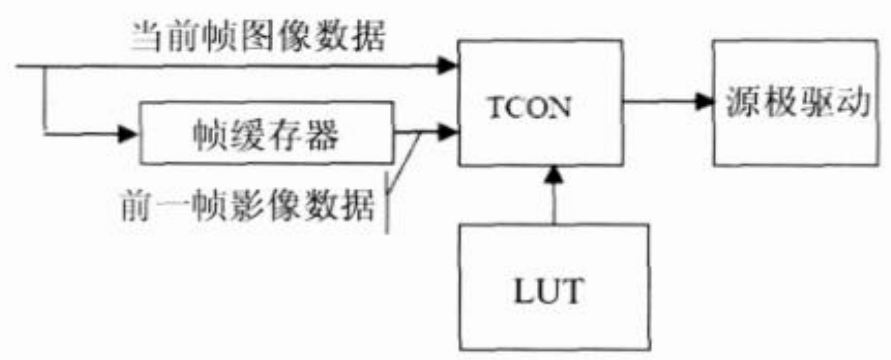

图 2. 过驱动系统结构框图

\section{过驱动下的液晶位相响应}

在液晶相位调制器中, $671 \mathrm{~nm}$ 的光可以实现0 4 $\pi$ 的相位调制。在未施加过驱动情况下, 相位 调制系统的上升响应时间和下降响应时间与位相的关系如图3、图4所示。

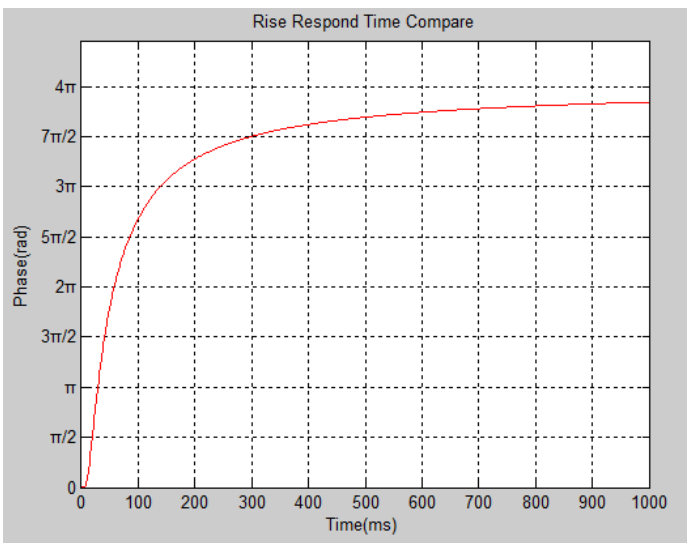

图 3.正常驱动电压下上升响应时间

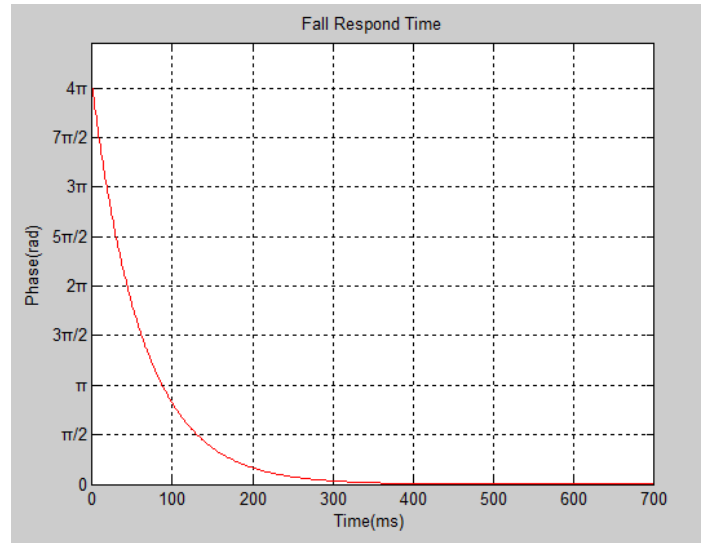

图 4.正常驱动下下降响应时间

可见在正常驱动电压的情况下，液晶的上升响应时间达到 $600 \mathrm{~ms}$ ，下降响应时间达到 $300 \mathrm{~ms}$, 严重影响液晶相位调制器的系统性能。根据液晶的动力学理论, 及液晶的弛豫特性, 在 0 到 $4 \pi$ 的位相范围选取合适的相位差为 $2 \pi$ 的相位段来实行液晶的过驱动。

如图 5 , 对在 $2 \mathrm{~V}$ 和 $5 \mathrm{~V}$ 驱动电压下液晶指向矢分布进行模拟，根据指向矢分布求得了各电压 下的位相。如图5所示，达到相同目标相位的情况下，5V驱动电压所用的上升时间远远少于 $2 \mathrm{~V}$ 驱动电压，且在 $5 \mathrm{~V}$ 驱动电压下液晶的 $2 \pi$ 上升响应时间缩短至 $30 \mathrm{~ms}$ 。

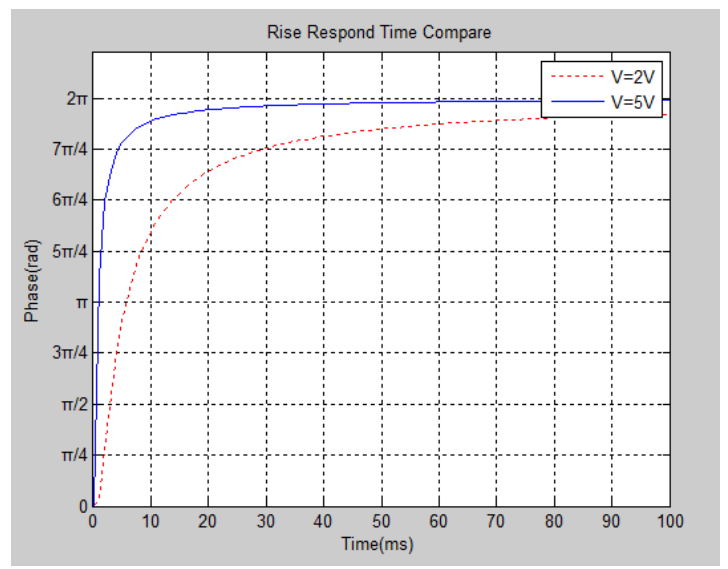

图5.不同驱动电压下上升响应时间对比

对液晶的响应下降过程, 将响应过程各时刻的指向矢分布转化为位相，仿真中驱动电压选 择从5V直接下降到 $0 \mathrm{~V}$,得到的下降位相响应曲线，如图6所示。 


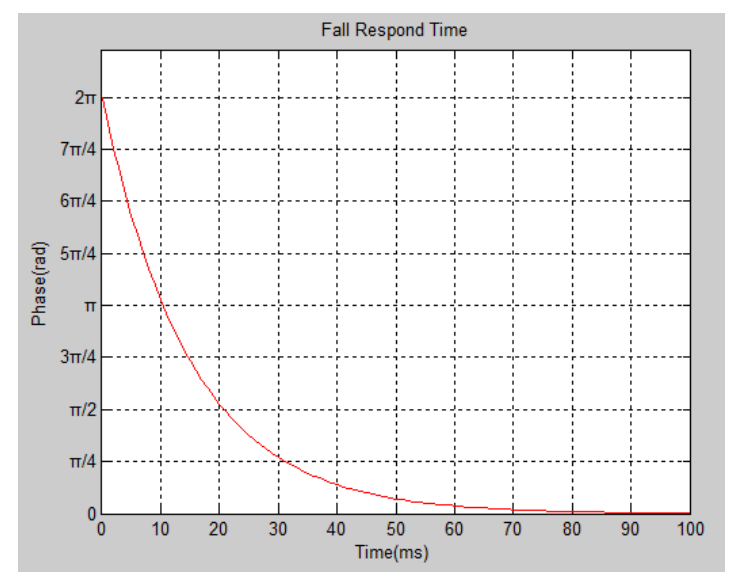

图6.下降响应时间

从图中可以看出直接撤去驱动电压后, 下降响应速度大大提高。 $2 \pi$ 下降响时间缩短至 $70 \mathrm{~ms}$ 。 证明过驱动可以实现液晶分子的快速偏转, 明显提高液晶空间光调制器的响应速度。

\section{结论}

本文通过分析液晶弛豫特性, 提出了运用过驱动提高液晶相位调制器响应速度的方案。通过 仿真, 选取合适的相位段, 验证了随着驱动电压的改变, 液晶位相变化明显, 在 $5 \mathrm{~V}$ 电压驱动 下 $2 \pi$ 上升响应时间缩短至 $30 \mathrm{~ms}, 2 \pi$ 下降响时间缩短至 $70 \mathrm{~ms}$, 证明过驱动技术可以很好的实现 液晶分子的快速偏转, 提高液晶相位调制器的响应速度。

\section{References}

[1] Jian Zhang,Yun Fang and Liying Wu:submitted to Chinese Journal of Lasers (2010). In Chinese.

[2] Wenben Xiao, the Research of Beam Deflection Control Technology Based on Liquid Crystal Optical Phase Array, edtied by D. Chinese Academy of Sciences Publising, Beijing (2013), in press.In Chinese.

[3] Xiangjie Zhao, Dayong Zhang and Yongquan Luo: submitted to Journal of High Power Laser and Particle Beams(2012). In Chinese.

[4] Ziqiang Huang: Principle of Liquid Crystal Display, edtied by M. National Defense Industry Press Publising, Beijing (2008). In Chinese.

[5] Xiangyi Nie, Haiqing Xianyu, et: submitted to Journal of Display Technology(2007)

[6] Junjun Gu, Rongyu Li and Yongliang Qiu: submitted to Journal of Modern Display(2008). In Chinese.

[7] Hongbin Hu, Lifa Hu,et: submitted to Journal of Opticsl Letters(2012) 